\begin{tabular}{ccc}
\hline International Journal of Engineering \& Technology, $7(4.30)(2018) 276-280$ \\
SPC \\
Website w ww. sciencepubco.com/index.php/IJET \\
Research paper
\end{tabular}

\title{
Brainwave Analysis for Robot Movement Depending on Age and Sex Differences
}

\author{
Norasyimah Sahat ${ }^{1}$, Afishah Alias ${ }^{2 *}$, Fouziah Md Yassin $^{3}$ \\ ${ }^{1,2}$ Faculty of Applied Science and Technology, Universiti Tun Hussein Onn Malaysia, Educational Hub Pagoh, 84600, Muar, Johor, \\ Malaysia \\ ${ }^{3}$ Faculty of Science and Natural Resources, Universiti Malaysia Sabah, 88400, Kota Kinabalu, Sabah, Malaysia \\ *Corresponding author E-mail: afishah79@gmail.com
}

\begin{abstract}
A Brain-Computer Interface (BCI) is a direct communication pathway between a human and external device. This system is very useful especially for disabled people as their brainwave still can emit electrical activity and can move the machine even with severe motor impairments. This research aims to investigate the brain waves produced by humans in terms of attention level for robot movement based on sex and age category of children (6-12 years), teenagers (18-25 years old) and adult (30 years and over). An Electroencephalography (EEG) device called Neurosky Mindwave Mobile has been used to obtain brainwave signals produced by humans. There were five aspects of robot movement namely forward (F), right (R), left (L), backward (B) and stop (S). From the analysis, the subject is less focus when doing the backward movement compared to another aspect of movements. Based on sex difference, the male has a higher attention level than female in every aspect of movement except for the left movement. The age group that has the highest attention level is teenager and the lowest is adult. It can be concluded that the attention level produced by human varies depending on age and sex difference of the individual itself.
\end{abstract}

Keywords: Attention level; Brain-Computer Interface; Brain wave; Neurosky Mindwave Mobile.

\section{Introduction}

In today's increasingly sophisticated technology, the creation of technology has influenced human life. The development integrated robot in order to be controlled by users has also been rapidly generated. Electric wheelchairs have also been built to help disabled people in their daily activities. However, this is especially limited to patients who have fully paralyzed or have a spinal cord injury that prevents them from controlling wheelchairs using a control device. In recent years, so much research has been done to produce controlled electric wheelchairs only by using brainwave signals through the Brain-Computer Interface (BCI) system. The BCI system is especially useful for disabled people or paraplegic patients.

Brain-Computer Interface (BCI) is a system that uses communication networks between a human brain nervous system and machine or computer. Through this system, people can move machines or computers without using their limbs and this system is especially useful for disabled people such as paralysis and stroke [1].

Brain Computing is a technology that makes human brainwaves as an input and will be accessed to produce output. Humans can send signals to an object or mechanism by using only the force of mind or rather the brainwave. Brainwaves can be detected using sensors placed on the scalp. The mental states and condition of the person can be configured by determining the brainwave type that being produced by that person. Analog brainwave signal has been converted into digital form to measure the speed and attention. This measurement and recording process known as Electroencephalography (EEG) [2].

This research has been studied to analyze the human brainwave pattern produced by humans depending on age and gender category by using Neurosky Mindwave Mobile in terms of attention level (related to beta wave) to improve the quality of signal and the accuracy of wheelchair robot's movement based on the result obtained.

\section{Related works}

\subsection{Brain-Computer Interface (BCI)}

$\mathrm{BCI}$ is a technology that allows people to control a mechanism using only the power of mind. This technology provides communication and control capabilities to people with severe motor disabilities. This can be done by placing the electrode on the head to detect the signal or more accurately the brain waves generated and then sent to the computer to analyze the information [3].

The BCI study objective is to restore vital functions to people who are highly disabled by various neuromuscular disorders, and enhance the functioning of healthy individuals [4]. The basic concept of BCI consists of five steps; signal acquisition, signal enhancement, feature extraction, classification and control interface. Figure 1 shows the general concept used for the BCI system [5]. 


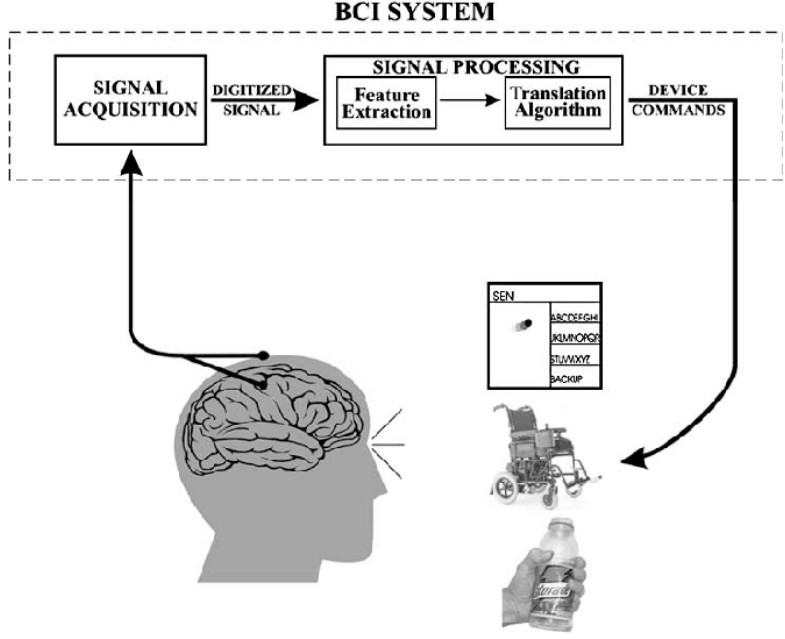

Fig.1: General concept of BCI

\subsection{Electroencephalography (EEG)}

Electroencephalography (EEG) is the first non-invasive neuron imaging technique used to measure brain electrical activity. EEG signals are detected from the scalp and contain noise due to electrical disturbance and electrode movement. The EEG measures brain waves (voltage graphs over time) through electrodes by using the sum of potential actions transmitted by neurons in the brain. An electroencephalogram is a record of the oscillations of brain electric potential recorded from electrodes positioned on the human scalp [6]. EEG is usually recorded by using common standard called International 10-20 system as shown in Figure 2. This system is an internationally recognized method to describe and apply the location of scalp electrodes in the context of an EEG experiment [7].

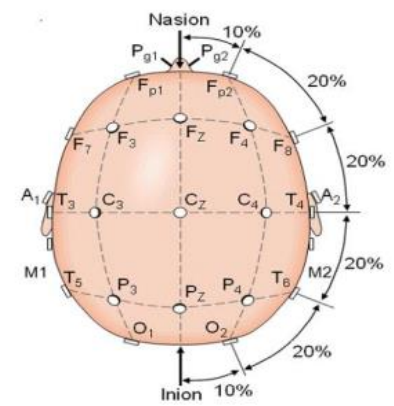

Fig. 2: 10/20 Electrode Positioning System

\subsection{Characteristics of the brainwave}

Figure 3 shows the types of brain waves produced by humans. Each brainwave has its own state of affairs.

\begin{tabular}{|c|c|c|}
\hline Brainwave Type & Frequency range & Mental states and condditions \\
\hline Delta & $0.1 \mathrm{~Hz}$ to $3 \mathrm{~Hz}$ & $\begin{array}{l}\text { Deep, dreamless sleep, non-REM sleep, } \\
\text { unconscious }\end{array}$ \\
\hline Theta & $4 \mathrm{~Hz}$ to $7 \mathrm{~Hz}$ & $\begin{array}{l}\text { Intuitive, creative, recall, fantasy, } \\
\text { imaginary, dream }\end{array}$ \\
\hline Alpha & $8 \mathrm{~Hz}$ to $12 \mathrm{~Hz}$ & $\begin{array}{l}\text { Relaxed, but not drowsy, tranquil, } \\
\text { conscious }\end{array}$ \\
\hline Low Beta & $12 \mathrm{~Hz}$ to $15 \mathrm{~Hz}$ & $\begin{array}{l}\text { Formerly SMR, relaxed yet focused, } \\
\text { integrated }\end{array}$ \\
\hline Midrange Beta & $16 \mathrm{~Hz}$ to $20 \mathrm{~Hz}$ & Thinking, aware of self \& surroundings \\
\hline High Beta & $21 \mathrm{~Hz}$ to $30 \mathrm{~Hz}$ & Alertness, agitation \\
\hline Gamma & $30 \mathrm{~Hz}$ to $100 \mathrm{~Hz}$ & Motor Functions, higher mental activity \\
\hline
\end{tabular}

Fig. 3: Brainwave type
Delta wave $(0 \mathrm{~Hz}-3 \mathrm{~Hz})$

This wave has a very large amplitude. This is a stage where people are asleep and unconscious. Humans are currently in a state of sleep without a dream. Studies show that this wave will decrease as the age increases. Often patients with brain disorders such as coma and bleeding often produce this wave [8].

Theta wave $(4 \mathrm{~Hz}-7 \mathrm{~Hz})$

These waves often produce much in children. The waves show that the brain is in full stage of relaxation as practiced by yoga masters. Humans who experience this wave are always in fantasy, have a lot of creative imaginations or minds [9].

Alpha wave $(8 \mathrm{~Hz}-12 \mathrm{~Hz})$

This is the level of human feeling in rest, meditating or meditation. This wave shows that humans are in a state of calm and are in a state of conscious mind that is conscious and unconscious. Often the decisions taken while in this situation are most appropriate in solving a problem [10].

Beta wave $(13 \mathrm{~Hz}-40 \mathrm{~Hz})$

This wave is a conscious mind. Usually occurs in Parietal Lobe and Frontal Lobe. The beta wave can be divided into three parts: low beta $(12-15 \mathrm{~Hz})$, midrange beta $(16-20 \mathrm{~Hz})$ and high beta $(21-$ $30 \mathrm{~Hz}$ ). Low beta show that the human being is in focus but still in a state of relaxation. For the midrange beta, people who experience this wave condition perform thought, focus and mindfulness. High beta waves are also a ranking where the brain is in a state of alert and has turbulence in itself [11].

\subsection{Neurosky Mindwave Mobile}

Mindwave Mobile (Figure 4) is a brainwave detection device used to capture signals in the brain and to translate it into visual or digital form. This tool has a function such as eSense. ESense is a specialized Neurosky patent algorithm in classifying existing brain waves. There are two types of eSense; eSense Attention and eSense Meditation. eSense Attention meter shows the concentration and focus of Mindwave Mobile users. Disturbances, drifting thoughts, less focus or anxiety can lower the level of the person's attention. The eSense Meditation meter shows the user's "tranquil" level. According to an explanation from Neurosky, brain waves related to concentration are beta waves while those relating to peace of mind are alpha waves. The waves were measured using a unique scale value set by Neurosky between 0 and 100 [12].

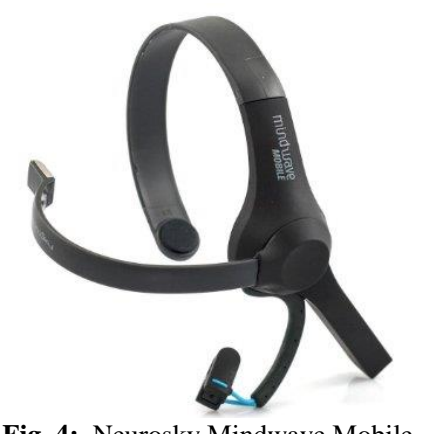

Fig. 4: Neurosky Mindwave Mobile

\section{Methodology}

\subsection{Block diagram of brainwave detection system}

Figure 5 shows the block diagram of the brainwave detection system. The brainwave signal generated by the user will be tracked by Mindwave Mobile and transmitted to the Arduino UNO microcontroller via wireless network. The Arduino UNO will send instructions to the servo motor to move the robot forward, right, left, 
backward and stop depending on the Mindwave Mobile signal. After that, the EEG signal of a user based on the attention level will be analyzed for controlling the robot movement. Arduino Integrated Development Environment (IDE) software has been used to get the attention value in a digital form ranging from 0 to 100 by using code provided by Neurosky. The attention value being analyzed based on age and gender category for every aspect of movement.
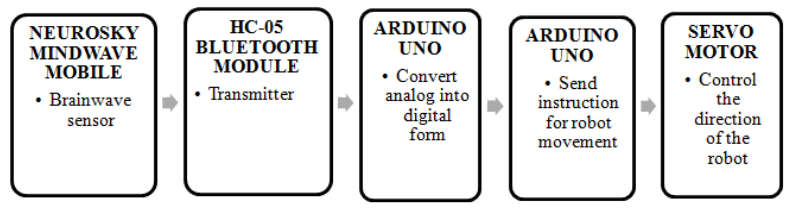

Fig.5: Block diagram of brainwave detection system [13]

\subsection{Experimental procedure}

A total of 50 respondents according to gender and age category which is; 20 children (6-12 years old), 20 teenagers (18-25 years old) and 10 adults (30 years and over) were selected for the brainwave analysis experiment for controlling the integrated robot movement. There are five aspects of robot movements to be taken into account, namely forward (F), right (R), left (L), backward (B) and stop (S). Figure 6 shows the flow diagram of the experiment conducted to a user for each type of age and gender category.
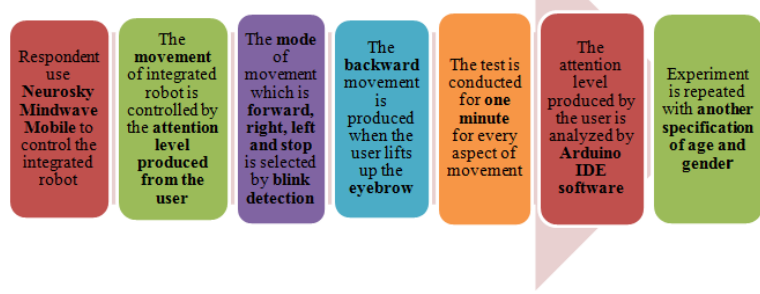

Fig 6: Experiment flow diagram of Mindwave Mobile users [14]

The tests are conducted according to the following steps:

1. Respondent is required to use Neurosky Mindwave Mobile to control the movement of the integrated robot.

2. User is directed to highly focus as the robot will move when the user raises the attention level over the threshold value.

3. Mode of movement which is forward (F), right (R), left $(\mathrm{L})$ and stop $(\mathrm{S})$ is selected by the blink detection produced by the user.

4. Backward (B) movement is selected by when the user lifts up the eyebrow as the poor signal quality is produced.

5. Experiment is conducted for one minute for every aspect of the movement.

6. The resulting concentration level is analyzed by using the Arduino IDE software.

7. Steps 1 until 6 are repeated using another specification of age and gender.

\section{Results and Discussion}

\subsection{Range of attention value}

Figure 7 shows that the attention level of the children's stage for males is higher than female except for backward movement. The males have great attention (80-100) during the forward and right movements and hard to focus when doing the backward movement compared to the females as the maximum range of attention value is $50-60$.
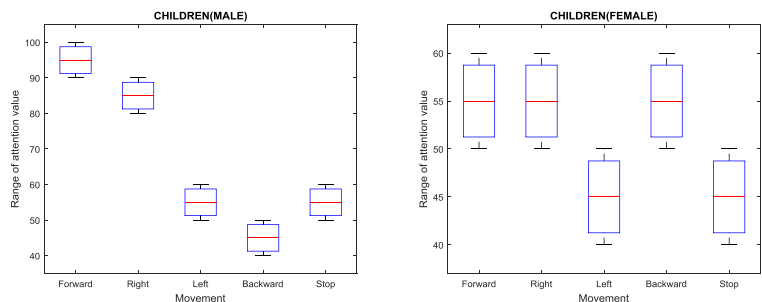

Fig. 7: Range of attention value for the children

The level of attention produced by the teenager's level of sex differences is shown in Figure 8. The data show that for the teenager category, females are more focused than males in all robot movement except during left and stop movement. The range of attention levels produced by teenage girls is between 50 and 60 which classified as neutral attention.
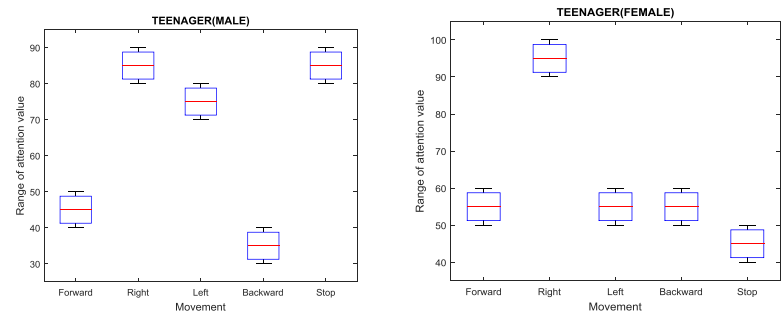

Fig. 8: Range of attention value for the teenager

Figure 9 shows that the maximum level of attention level for the adult stage is during the right movement of $90-100$. Based on the gender comparison, male adults have higher levels of attention than women for each component of the movement. It also shows that during adult stage, the level of attention that can be produced is very less compared to another category of age.
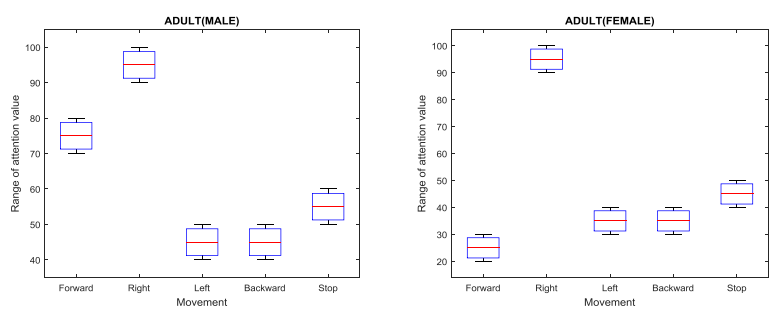

Fig. 9: Range of attention value for the adult

\subsection{Average of maximum attention value}

Figure 10 shows that for forward movement, the teenager has the highest attention value compared to another age category. Women's adult has the lowest average of maximum attention level when doing a forward movement of 68.6. In terms of gender, men are more focused than women for each age category when doing forward movement.

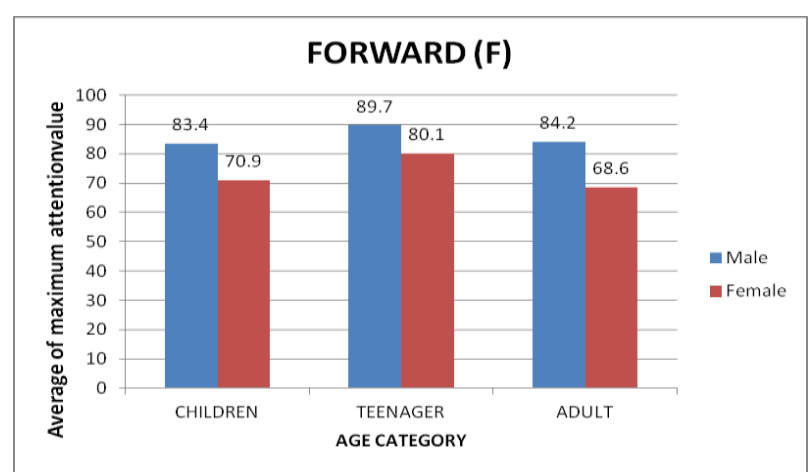

Fig. 10: Average of maximum attention value for forward movement 
From Figure 11, it shows that teenager has the highest attention value for right movement and the lowest is children. For the right mode of robot movement, males have the highest attention level compared to females.

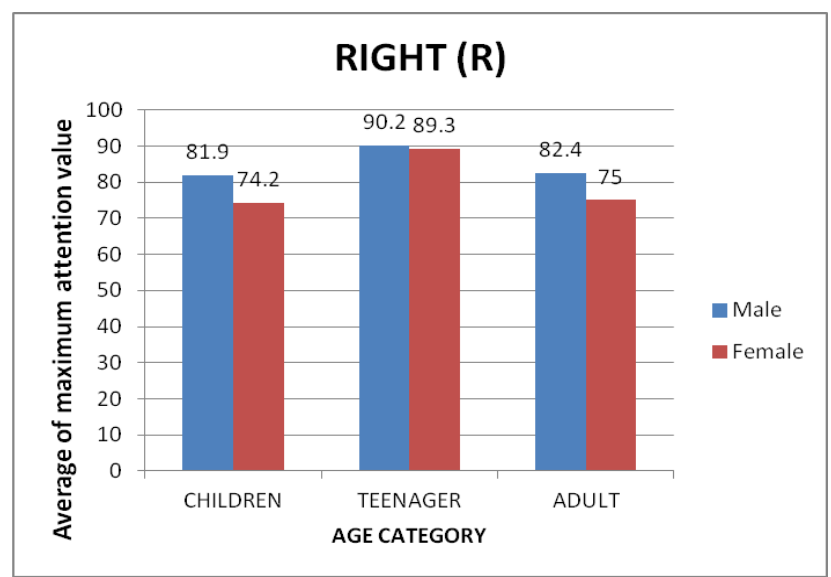

Fig. 11: Average of maximum attention value for right movement

The female's attention values for all age category when doing left movement is higher than males as shown in Figure 12. Based on age comparison, the teenager has the highest attention value while the adult has the lowest.

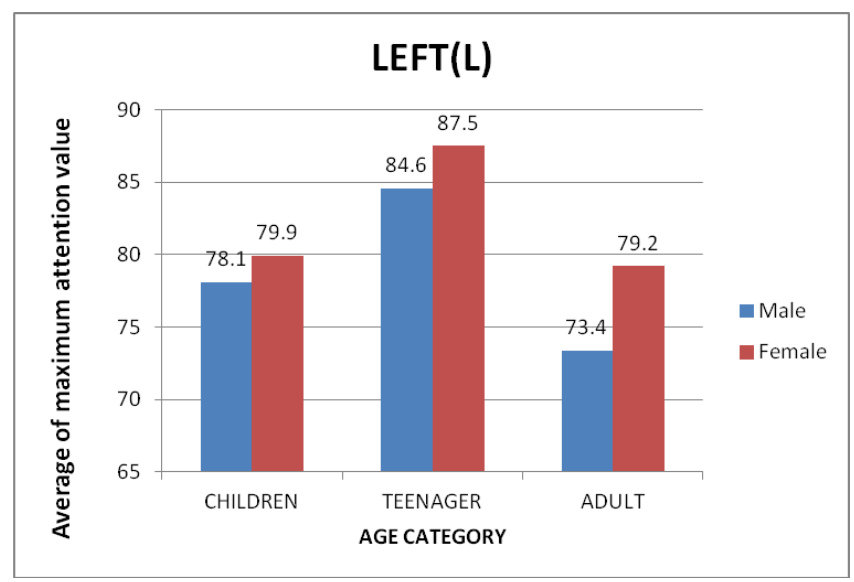

Fig. 12: Average of maximum attention value for left movement

From Figure 13, males have the higher attention level than females for all type of category. The highest attention value produced is in the teenager stage while only slightly different of attention value between children and adult.

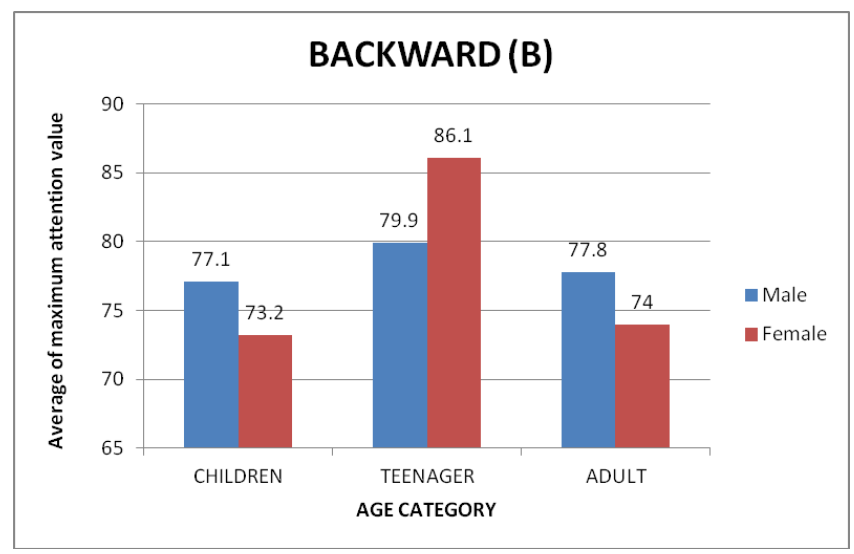

Fig. 13: Average of maximum attention value for backward movement

Figure 14 shows that the teenager has the highest attention value among the age groups while the adult and children has slightly different of attention value but the lowest attention value for stopping the robot movement is in children stage.

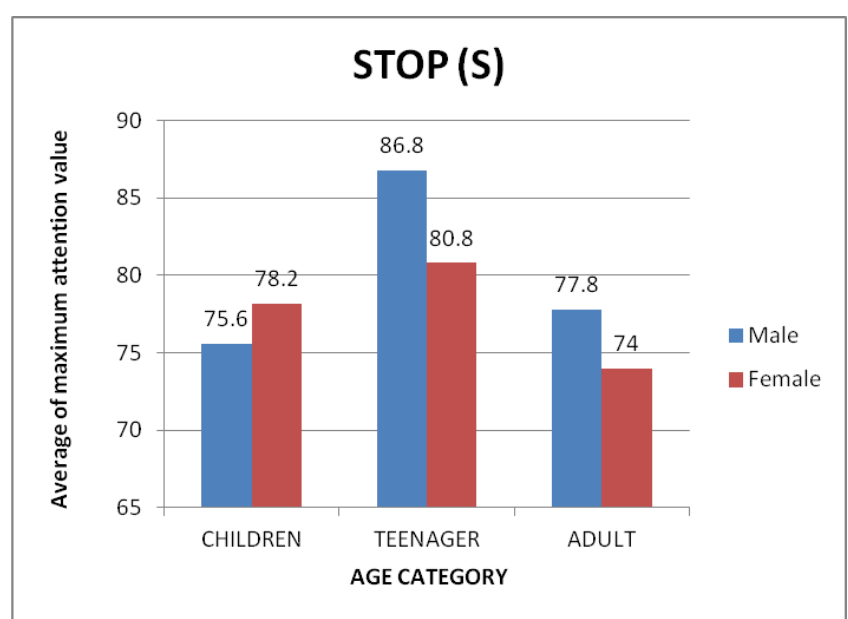

Fig. 14: Average of maximum attention value for stop movement

\subsection{Age and sex differences in attention}

The percentage of the average maximum attention value for each gender ( 25 males and 25 females) for every aspect of movement regardless of the age category has been calculated as shown in Figure 15. The overall percentage for the average maximum attention value can be calculated by the total average of maximum attention value divided by the number of participants. It shows that for every aspect of movement, people tend to focus more when doing right movement while the lowest attention value is when doing backward movement. In gender category, the attention value produced by male is higher than female.

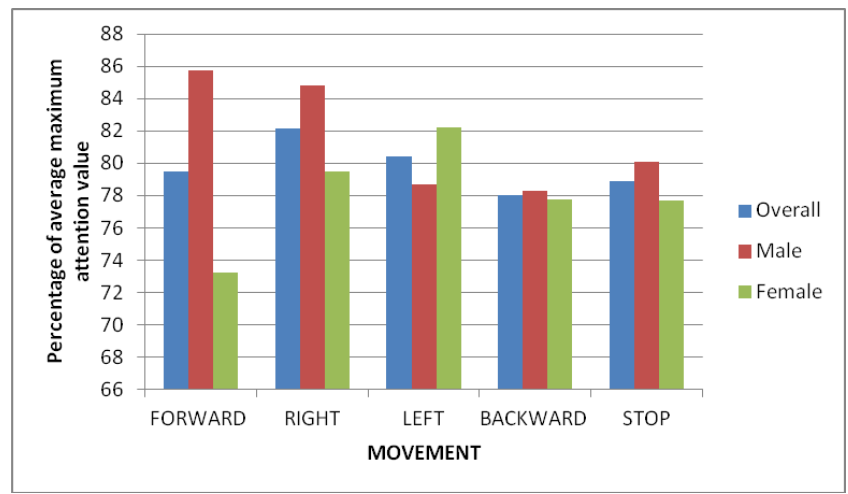

Fig.15: Percentage of the maximum attention value

Previous study shows that there are various factors that can affect the human attention value and some of the effects is based on the age and sex differences of the individual [15]. However, the analysis of attention value based on gender and age category is not being fixed as each human brain is different and another factor such as surrounding and disease factors associated with the human brain also can lead to less attention.

In this research, an EEG device called Neurosky Mindwave Mobile has been used to acquire the brainwave data produced by the human. EEG is the first non-invasive method. The dry type of EEG used an active electrode to record the EEG data at the fp1 position located on the frontal lobe with reference to the earlobe. Hans Berger states that beta activity is associated with the metabolic process of cortical tissue and related to the attention. The higher the amount of beta wave produces, the higher the level of attention [16].

Through experiments conducted show that male is more likely to focus than female for all aspects of movement except for the left movement where the level of women's attention is higher than 
men. The processing of getting the raw data of brainwave is located in the frontal lobe of the brain for EEG system as the cognitive function has commonly associated with the prefrontal cortex. A research based on the brain anatomy found that the size of the frontal lobe in the male is $1 \%$ larger than that of the female [17] Besides that, male are more focus on in specific task and less sensitive to the surrounding compared to the female [18].

However, the results obtained during the left movement are very different where the female has higher levels of attention than male. This result is really significant to the investigation study of the attention level of undergraduate students based on gender differences [19]. The results show that female students have a better ability to pay attention than male students. Female students pay a higher level of attention during visual-based activities while male students are good at hand-based activities. Gray matter is really important in attention because this area contains many nerve cells or neurons. Based on brain anatomy, women have a higher percentage of gray matter while men have a higher percentage of white matter [20]. However, female is more sensitive about emotion which can affects the resulting concentration [21].

Analysis of brainwave signals shown that a teenager has the highest attention level followed by children and adult has the lowest attention value in every aspect of the movement. A teenager has the highest attention value because beta waves produced is higher as most people having so much mental activity at this age and more emotion or mental states happen in this age life span.

Research based on the brain anatomy concluded that the frontal volume of the human varies according to age. As we know, the collection of EEG signals is located in the frontal lobe of the human brain. Older subject has less frontal lobe volume compare to the younger subject. That's why the adult has the lowest attention value than child and teenager. Besides that, differences in cognitive abilities among age category can be related to some disease occur that affect the human attention such as attention deficit disorder (ADD), especially for children. According to statistic, ADD is affecting $3 \%$ to $10 \%$ of children and $4 \%$ of the adult [22].

For aspect of movements, the right mode of movement has the highest attention level compared to another aspect of the movement. The lowest of attention level produced by the human is when doing the backward movement. This is due to the difficult task to imagine the robot to reverse instead of going forward when focus. Therefore, eyebrow movement is being used for backward movement instead of using attention level.

\section{Conclusion}

Based on the experiment, it can be concluded that age and sex differences could affect the attention level produced by the humans. From the analysis, male is easier to focus compared to the female for every aspect of robot movement except for left movement. Based on age comparison, the teenager has the highest attention value followed by children while the adult has the lowest attention value in all age category studied. Since the attention value for every aspect of the movement varies according to age and sex differences, the threshold value for the integrated robot movement controlled by Neurosky Mindwave Mobile can be set differently based on the age and sex category of the subject.

\section{Acknowledgement}

The author is grateful to Universiti Tun Hussein Onn Malaysia and Universiti Malaysia Sabah for providing relevant instruments in conducting this research. This work was funded by Research Management Centre, Universiti Tun Hussein Onn Malaysia under TIER 1 (H066) Research Grant.

\section{References}

[1] Devi MA, Sharmila R \& Saranya V (2014), Hybrid brain computer interface in wheelchair using voice recognition sensors. International Conference on Computer Communication and Informatics (ICCI), 1-5.

[2] Ismail WW, Hanif M, Mohamed SB, Hamzah N \& Rizman ZS (2016), Human emotion detection via brain waves study by using electroencephalogram (EEG), International Journal on Advanced Science, Engineering and Information Technology 6(6), 1005-1011.

[3] Leuthard EC, Schalk G, Moran DW, Wolpaw JR \& Ojemann JG, U. S. Patent 7(120), 486, (2006)

[4] Mor M \& Juvvala L, Brain computer interface (2018), $3^{\text {rd }}$ International Conference on Computing: Communication, Networks and Security (IC3NS) 4(3), 30-34.

[5] Ramesh S, Krishna MG \& Nakirekanti M (2014), Brain computer interface system for mind controlled robot using Bluetooth. International Journal of Computer Applications 104(15), 20-23.

[6] Jayabhavani GN, Raajan NR \& Rubini R (2013), Brain mobile interfacing (BMI) system embedded with wheelchair, Proceeding of IEEE Conference on Information and communication technologies (ICT), 1129-1133.

[7] Birbaumer N (2006), Breaking the silence: brain-computer interfaces (BCI) for communication and motor control, Psychophysiology 43(6), 517-532.

[8] Rao RPN, Brain-Computer Interfacing: An Introduction, University of Washington. Cambridge: Cambridge University Press, (2013).

[9] Arora R \& Bhattacharyya S (2014), An approach towards brain actuated control in the field of robotics using eeg signals: a review, International conference of Advance Research and Innovation (ICARI), 103-113.

[10] Rahman KAA, Ibrahim KK, Salam B, Huq MS, Nasir NHM, Ahmad MKI \& Sherwani F (2015), Graphical user interface controlled via brainwave signals for paraplegic rehabilitation. International Conference on Electrical and Electronic Engineering.

[11] Akila M, Sekar KS \& Suresh A (2015), Smart brain-controlled wheelchair and devices based on EEG in low cost for disabled person. International Journal of Computers Communication Networks and Circuit Systems 1(1), 291-298.

[12] Neurosky Inc (Ed.), NeuroSky's eSenseTM Meters and Detection of Mental State, (2009).

[13] Yassin MDF, Apin D, Abd Rahman AB \& Alias A (2017), Multimode Brainwave Controller. Advanced Science Letters 23(11), 11508-11511.

[14] Yassin MDF, Sahat N, Chin SN \& Alias A (2018), The brain wave analysis for robot movement using one electrode. Academic Jour nal of Science (AJS) 8(1), 15-22.

[15] Li C \& Shallcross DJ (1992), The effect of the assumed boundary in the solving of the nine-dot problem on a sample of Chinese and American students 6-18 years old. The Journal of Creative Behaviour 26 (1), 53-64.

[16] Vallabhaneni A, Wang $\mathrm{T} \& \mathrm{He} \mathrm{B}$, Brain-computer interface in Neural Engineering, Springer, (2005), 85-121.

[17] Swaab DF \& Man H (1984), A historical perspective: Sex differences in the brain. The Relation Between Structure and Function 61 361.

[18] Holzel BK, Carmody J, Vangel M et al. (2011), Mindfulness practice leads to increases in regional brain gray matter density. Psychiatry Research: Neuroimaging, 191(1), 36-43.

[19] Kan DPX, Lim VWW \& Lee PF (2015), Signal conversion from attention signals to light emitting diodes as an attention level indicator. In $1^{\text {st }}$ Global Conference on Biomedical Engineering and $9^{\text {th }}$ Asian-Pasific Conference on Medical and Biological Engineering, 251-255.

[20] Gur RC, Turetsky BI, Matsui M et al. (1999), Sex differences in brain gray and white matter in healthy young adults: correlations with cognitive performance. Journal of Neuroscience 19(10), 40654072.

[21] Tun PA \& Lachman ME (2008), Age differences in reaction time and attention in a national telephone sample of adults: education, sex and task complexity matter. Developmental psychology 44(5), 1421.

[22] Ardeshiri A, Wenger E, Holtmannspotter M \& Winkler PA (2006), Surgery of the anterior part of the frontal lobe and of the central region: normative morphometric data based on magnetic resonance imaging. Neurosurgical review 29(4), 313-321. 